\title{
Behavioral Analysis of Dopaminergic Activation in Zebrafish and Rats Reveals Similar Phenotypes
}

Fredrik $\mathrm{Ek}^{\mathrm{a}}$, Marcus Malo ${ }^{\mathrm{b}}$, Madelene Åberg Andersson ${ }^{\mathrm{a}}$, Christoffer Wedding ${ }^{\mathrm{a}}$, Joel Kronborg ${ }^{\mathrm{a}}$, Peder Svensson $^{\mathrm{c}}$, Susanna Waters ${ }^{\mathrm{c}, \mathrm{d}}$, Per Petersson ${ }^{\mathrm{e}}$, and Roger Olsson ${ }^{\mathrm{a}}$

\section{Supporting information}

Sequence alignment

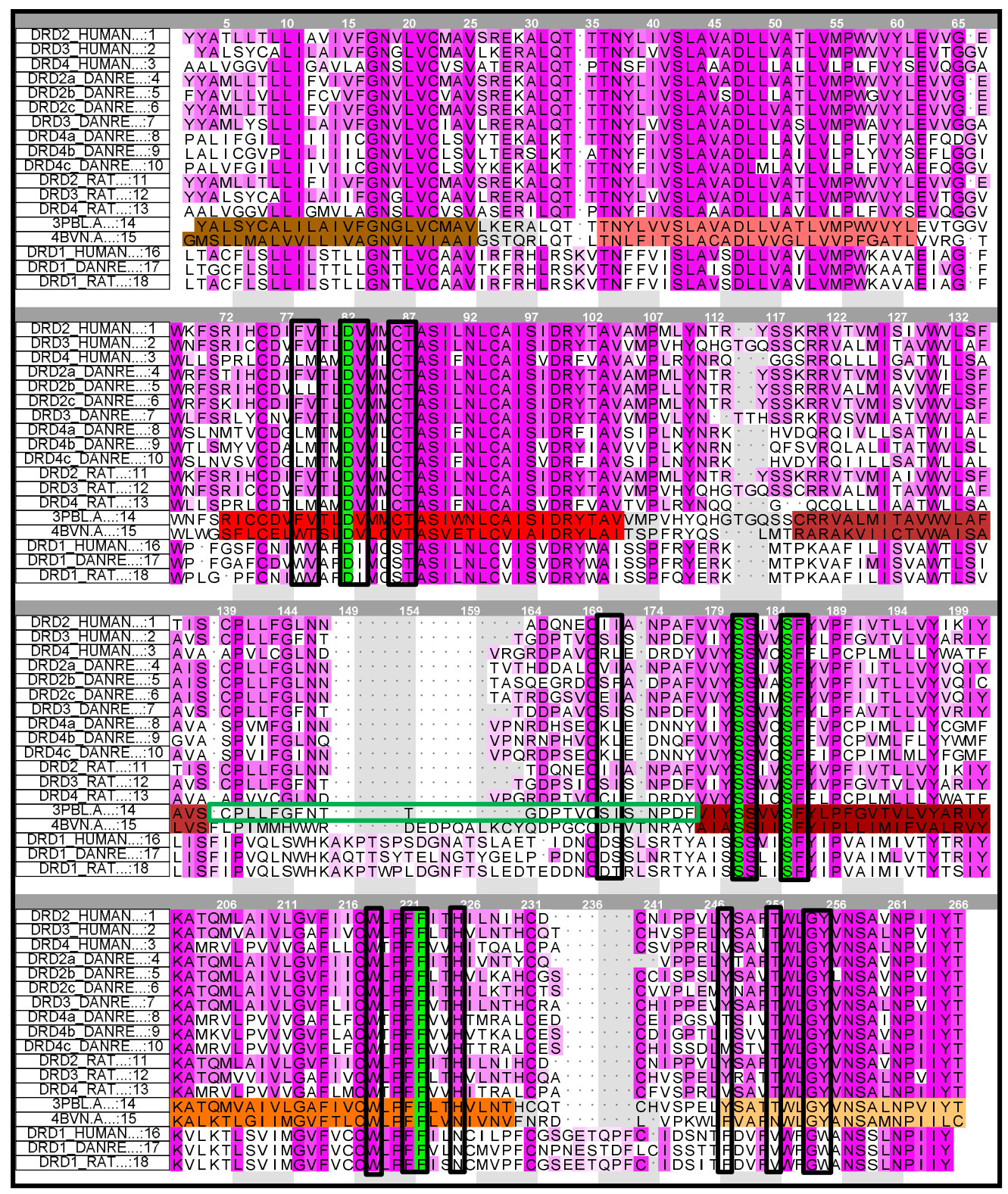


Figur 1s. A refined multiple alignment including a selection of human, zebrafish and rat dopamine receptor sequences. Dark purple indicate conserved amino acids, while light purple indicate less conserved but still similar amino acids. The sequences used as template in the receptor modelling, the turkey adrenergic $\beta_{1}(4 \mathrm{BVN})$ and the human dopamine $\mathrm{D}_{3}$ receptor structure (3PBL) are also included. The transmembrane regions in the crystal structures are highlight with seven boxes (TM1-7). The N-and C-terminus have been excised as well as the third intracellular loop, between TM5 and TM6. The second extracellular loop (EC2) which is important for ligand binding and used as template in the modeling of the $\mathrm{D}_{2}$-like dopamine receptors is highlighted with a green box. The residues in the black boxes are those within $4 \AA$ from apomorphine in the zebrafish dopamine $\mathrm{D}_{2}$ receptor model (drd2a_danre) and the green highlighted are the agonist key amino acids.

Table 1s. Sequence similarity based on the complete alignment above

\begin{tabular}{|c|c|c|c|c|c|c|c|c|c|c|c|c|c|c|c|c|c|c|}
\hline & 1 & 2 & 3 & 4 & 5 & 6 & 7 & 8 & 9 & 10 & 11 & 12 & 13 & 14 & 15 & 16 & 17 & 18 \\
\hline 1:DRD2_HUMAN... & & 72.1 & 49.2 & 89.5 & 79.2 & 88.3 & 76.2 & 49.6 & 50.8 & 49.2 & 97.9 & 71.4 & 48.3 & 71.7 & 39.7 & $37.7 \mid$ & 37.2 & 37.6 \\
\hline 2:DRD3_HUMAN... & 73.3 & & 51.3 & 72.6 & 70.0 & 71.7 & 82.0 & 47.1 & 50.4 & 47.9 & 73.3 & 95.9 & 50.4 & 99.2 & 37.7 & 35.8 & 36.8 & 34.5 \\
\hline 3:DRD4_HUMAN... & 50.0 & 51.3 & & 50.2 & 52.9 & 49.6 & 53.6 & 66.7 & 69.2 & 68.3 & 58.8 & 58.2 & 90.8 & 51.3 & 35.6 & 34.2 & 34.5 & 33.7 \\
\hline 4:DRD2a_DANR... & 89.8 & 71.7 & 49.6 & & 79.2 & 92.1 & 75.7 & 49.2 & 48.8 & 48.3 & 89.8 & 71.4 & 48.3 & 70.8 & 48.9 & 37.7 & 37.6 & 38.0 \\
\hline 5:DRD2b_DANR... & 88.5 & 70.8 & 52.9 & 80.2 & & 81.3 & 72.8 & 49.6 & 53.3 & 50.4 & 81.4 & 69.3 & 51.7 & 69.6 & 36.4 & 37.0 & 36.4 & 36.4 \\
\hline 6:DRDZC_DANR.. & 89.8 & 71.7 & 49.6 & 93.2 & 81.3 & & 76.2 & 50.0 & 50.4 & 49.6 & 89.8 & 71.4 & 48.7 & 78.8 & 39.7 & 38.9 & 37.6 & 38.4 \\
\hline 7:DRD3_DANRE... & 77.1 & 81.7 & 53.3 & 76.4 & 71.7 & 75.8 & & 50.0 & 51.7 & 49.2 & 78.0 & 80.5 & 50.8 & 80.8 & 48.9 & \begin{tabular}{|c|}
39.7 \\
\end{tabular} & \begin{tabular}{|c|}
39.9 \\
\end{tabular} & 38.4 \\
\hline 8:DRD4a_DANR... & 50.4 & 47.1 & 66.7 & 49.8 & 49.6 & 58.8 & 50.2 & & 72.1 & 87.9 & 58.4 & 47.7 & 67.6 & 47.1 & 32.8 & \begin{tabular}{|l|}
34.6 \\
\end{tabular} & 33.7 & 34.9 \\
\hline 9:DRD4b_DANR... & 51.7 & 58.4 & 69.2 & 49.4 & 53.3 & 50.4 & 51.9 & 72.1 & & 72.9 & 52.1 & 50.2 & 68.5 & 58.0 & 32.8 & 36.2 & 36.4 & 36.0 \\
\hline :DRDAC_DANR... & 50.8 & 47.9 & 68.3 & 48.9 & 50.4 & 49.6 & 49.4 & 87.9 & 72.9 & & 49.2 & 47.7 & 70.2 & 47.9 & 31.6 & 37.4 & 36.4 & 36.8 \\
\hline 11 :DRD2_RAT|P. & 97.9 & 72.1 & 49.2 & 89.5 & 80.0 & 88.3 & 77.0 & 49.6 & 51.3 & 48.3 & & 71.4 & 48.7 & 71.7 & 39.7 & \begin{tabular}{|l|}
$\mid 37.7$ \\
\end{tabular} & 36.8 & 37.6 \\
\hline 12:DRD3_RAT|P... & 72.9 & 96.3 & 50.4 & 72.6 & 69.6 & 71.7 & 81.2 & 47.9 & 58.4 & 47.9 & 72.9 & & 49.2 & 95.4 & 37.7 & 36.6 & 36.8 & 35.3 \\
\hline 13:DRD4_RAT|P... & 48.7 & 50.0 & 90.0 & 48.5 & 51.3 & 48.3 & 50.6 & 67.1 & 67.9 & 69.6 & 49.2 & 48.5 & & 50.8 & 35.6 & 34.2 & 34.9 & 34.1 \\
\hline 14:3PBL.A & 72.9 & 99.2 & 51.3 & $71.7 \mid$ & 69.6 & 70.8 & 81.2 & 47.1 & 50.0 & 47.9 & 72.9 & $95.0 \mid$ & 50.4 & & 37.7 & 35.4 & 36.8 & 34.1 \\
\hline 15:4BVN.A & 41.5 & 38.8 & $|36.7|$ & 42.6 & 37.5 & 40.8 & 42.3 & 32.9 & 32.9 & 32.5 & 41.5 & 38.6 & 37.8 & 38.8 & & 40.5 & 41.5 & 39.9 \\
\hline 16:DRD1_HUMAN... & 41.1 & 38.3 & $|36.7|$ & 48.9 & 39.6 & 41.7 & 42.7 & 37.1 & 38.8 & 48.8 & 41.1 & 39.8 & 37.0 & 37.9 & 42.1 & & 82.6 & 92.2 \\
\hline 17:DRD1_DANRE. . & 40.7 & 39.6 & 37.1 & \begin{tabular}{|c|}
48.9 \\
\end{tabular} & 39.2 & 48.4 & 43.1 & 36.3 & 39.2 & 39.2 & 48.3 & \begin{tabular}{|l|}
39.4 \\
\end{tabular} & 37.8 & \begin{tabular}{|c|}
39.6 \\
\end{tabular} & 43.3 & \begin{tabular}{|c|}
82.9 \\
\end{tabular} & & 81.0 \\
\hline B:DRD1_RAT|P. & 41.1 & 37.1 & 36.3 & 41.4 & 39.2 & 41.3 & 41.4 & 37.5 & 38.8 & 39.6 & 41.1 & 37.8 & 37.0 & 36.7 & 41.7 & 92.6 & 81.0 & \\
\hline
\end{tabular}

Table 2s. Sequence similarity transmembrane (TM) region 


\begin{tabular}{|c|c|c|c|c|c|c|c|c|c|c|c|c|c|c|c|c|c|c|}
\hline & 1 & 2 & 3 & 4 & 5 & 6 & 7 & 8 & 9 & 10 & 11 & 12 & 13 & 14 & 15 & 16 & 17 & 18 \\
\hline :DRD2_HUMAN . . . & & 79.5 & 53.5 & 93.6 & 81.4 & 93.6 & 82.0 & 55.8 & 58.1 & 54.7 & 97.7 & 77.9 & 54.4 & 78.9 & 48.3 & 49.7 & 49.1 & 49.7 \\
\hline 2:DRO3_HUMAN. .. & 79.1 & & 55.2 & 77.9 & 76.7 & 77.9 & 84.3 & 54.7 & 55.8 & 52.3 & 79.1 & 96.5 & 53.2 & 99.4 & 44.8 & 46.8 & 46.8 & 45.6 \\
\hline 3:DRD4_HUMAN. & 53.5 & 55.6 & & 53.5 & 58.7 & 53.5 & 55.8 & 75.8 & 76.2 & $76.7 \mid$ & 53.5 & 54.1 & 93.6 & 55.6 & 40.1 & 42.1 & 42.7 & 41.5 \\
\hline 4:DRD2a_DANR. & 93.6 & 78.4 & 53.5 & & 82.6 & 97.1 & 82.0 & 57.0 & 56.4 & 55.2 & 93.6 & 77.3 & 54.4 & 77.8 & 49.4 & 50.9 & 50.3 & 58.9 \\
\hline 5:DRD26_DANR. & 81.4 & 77.2 & 58.7 & 82.6 & & 83.7 & 77.9 & 55.8 & 59.9 & 55.2 & 82.6 & 75.6 & 57.9 & 76.6 & 44.2 & 46.8 & 47.4 & 46.2 \\
\hline 6:DRD2C_DANR... & 93.6 & \begin{tabular}{|c|}
78.4 \\
\end{tabular} & 53.5 & 97.1 & 83.7 & & 82.0 & 56.4 & 57.6 & 55.2 & 93.6 & 77.3 & 54.4 & 77.8 & 48.3 & 58.9 & 50.3 & 58.9 \\
\hline 7:DRD3_DAYREE... & $82 . \theta$ & 84.8 & 55.8 & 82.0 & 77.9 & 82.0 & & 57.8 & 57.8 & 55.2 & 83.1 & 82.6 & 55.8 & 84.2 & 49.4 & 51.5 & 51.5 & 50.3 \\
\hline 8:DRD4a_DANR.... & 55.8 & 55.8 & 5.8 & 57.8 & 55.8 & 56.4 & 57.0 & & 79.1 & 98.7| & 55.8 & 55.2 & 77.2 & 55.8 & 39.8 & 43.3 & 42.7 & 43.3 \\
\hline 9:DRD4b_DANR. & 58.1 & 56.1 & 76.2 & 56.4 & 59.9 & 57.6 & 57.8 & 79.1 & & 79.1 & 58.7 & 56.4 & 75.4 & 55.6 & 40.1 & 45.6 & 46.2 & 45.8 \\
\hline DRDAC_DANR. & 54.7 & 52.6 & 76.7 & 55.2 & 55.2 & 55.2 & 55.2 & 96.7 & \begin{tabular}{|c|}
79.1 \\
\end{tabular} & & 53.5 & 52.3 & 78.4 & 52.6 & 38.4 & 45.0 & 43.9 & 44.4 \\
\hline 1 :DRD2_RAT|P. & 97.7 & 79.5 & 53.5 & 93.6 & 82.6 & 93.6 & 83.1 & 55.8 & \begin{tabular}{|c|}
58.7 \\
\end{tabular} & 53.5 & & 77.9 & 55.8 & 78.9 & 48.3 & 49.1 & 48.5 & 49.1 \\
\hline $2: D R D 3$ RAT $\mid P \ldots$ & 77.9 & 97.1 & 54.1 & 77.3 & 75.6 & 77.3 & 82.6 & 55.2 & 56.4 & 52.3 & 77.9 & & 52.0 & 96.5 & 44.8 & 47.4 & 47.4 & 46.2 \\
\hline $3:$ DRD4_RAT|P. & 54.1 & 53.2 & 93.0 & 54.1 & 57.6 & 54.1 & 54.7 & 76.7 & 75.8 & \begin{tabular}{|c|}
77.9 \\
\end{tabular} & 54.7 & 51.7 & & 53.2 & 39.5 & 42.7 & 43.3 & 42.7 \\
\hline 14:3PBL.A & 78.5 & 99.4 & 55.2 & 77.3 & 76.2 & 77.3 & \begin{tabular}{|c|}
83.7 \\
\end{tabular} & 54.7 & 55.2 & 52.3 & 78.5 & 95.9 & 53.2 & & 44.8 & 46.2 & 46.2 & 45.8 \\
\hline :4BVN.A & & 45.8 & 40.1 & \begin{tabular}{|l|}
49.4 \\
\end{tabular} & 44.2 & 48.3 & 49.4 & 39.8 & 40.1 & 38.4 & 48.3 & 44.8 & 39.8 & 45.8 & & 50.3 & 49.7 & 49.7 \\
\hline 16:DRD1_HUMAN. .. & \begin{tabular}{|l|}
49.4 \\
\end{tabular} & 46.8 & 41.9 & 50.6 & 46.5 & 50.6 & 51.2 & 43.0 & 45.3 & 44.8 & 48.8 & 47.1 & 42.7 & 46.2 & 50.0 & & 90.6 & 97.1 \\
\hline 17:DRD1_DANRE. & 48.8 & 46.8 & 42.4 & 50.8 & 47.1 & 58.8 & 51.2 & 42.4 & 45.9 & 43.6 & 48.3 & 47.1 & 43.3 & 46.2 & 49.4 & \begin{tabular}{|c|}
90.6 \\
\end{tabular} & & 91.2 \\
\hline DRD1_RAT|P & 49.4 & 45.6 & 41.3 & 50.6 & 45.9 & 58.6 & 58.0 & 43.0 & 44.8 & 44.2 & 48.8 & 45.9 & 42.7 & 45.8 & 49.4 & 97.1 & 91.2 & \\
\hline
\end{tabular}

Table 3s. Sequence similarity within $4 \AA$ from apomorphine defining the binding pocket (20 amino acids)

\begin{tabular}{|c|c|c|c|c|c|c|c|c|c|c|c|c|c|c|c|c|c|c|}
\hline & 1 & 2 & 3 & 4 & 5 & 6 & 7 & 8 & 9 & 10 & 11 & 12 & 13 & 14 & 15 & 16 & 17 & 18 \\
\hline 1:DRD2_HUMAN.... & & 95.0 & 75.0 & 95.0 & 80.0 & 95.0 & 95.0 & 75.0 & 75.0 & 75.0 & 100.0 & 95.0 & 75.0 & 95.0 & 60.0 & 55.0 & 55.0 & 55.0 \\
\hline 2:DRO3_HUMAN... & 95.0 & & 75.0 & 95.0 & 85.0 & 95.0 & 100.0 & 75.0 & 75.0 & 75.0 & 95.0 & 100.0 & 75.0 & 100.0 & 60.0 & 55.0 & 55.0 & 55.8 \\
\hline 3:DRD4_HUMAN... & 75.0 & 75.0 & & 75.0 & 80.0 & 75.0 & 75.0 & 90.0 & 90.6 & 90.0 & 75.8 & 75.0 & 95.0 & 75.0 & 60.0 & 50.0 & 50.0 & 50.0 \\
\hline 4:DRD2a_DANR... & 95.0 & 95.0 & 75.0 & & 80.0 & 95.8 & 95.0 & 75.0 & 75.0 & 75.0 & 95.8 & 95.0 & 75.0 & 95.0 & 60.0 & 55.8 & 55.0 & 55.0 \\
\hline 5:DRO2b_DANR... & 80.0 & 85.0 & 80.0 & 80.0 & & 80.0 & 85.0 & 80.0 & 80.0 & 80.0 & 80.0 & 85.0 & 80.0 & 85.0 & 65.0 & 50.0 & $50 . \theta$ & 50.0 \\
\hline $6: D R D 2 C$ DAANR $\ldots$ & 95.0 & 95.0 & 75.0 & 95.0 & 80.0 & & 95.0 & 75.0 & 75.0 & 75.0 & 95.0 & 95.0 & 75.0 & 95.0 & 60.0 & 55.0 & $55 . \theta$ & 55.0 \\
\hline $7:$ DRD3_DANRE $\ldots$ & 95.0 & $100 . \ominus$ & 75.0 & 95.0 & 85.0 & 95.0 & & 75.0 & 75.0 & 75.0 & 95.0 & $\mid 100 . \ominus$ & 75.0 & 100.0 & 60.0 & 55.0 & $55 . \theta$ & 55.8 \\
\hline 8:DRD4a_DANR... & 75.0 & 75.0 & 90.0 & 75.0 & 80.0 & 75.0 & 75.0 & & 95.0 & 95.0 & 75.0 & 75.0 & 90.0 & 75.0 & 60.0 & 50.0 & 50.0 & 50.0 \\
\hline 9:DRD4b_DANR... & 75.0 & 75.0 & 90.0 & 75.0 & 80.0 & 75.6 & 75.0 & 95.0 & & 95.0 & 75.0 & 75.0 & 90.0 & $75 . \theta$ & 60.0 & 50.0 & 50.0 & 50.0 \\
\hline $10:$ DRD 4 C_DANR.... & 75.0 & 75.0 & 90.0 & 75.0 & 80.0 & 75.0 & 75.0 & 95.0 & 95.0 & & 75.0 & 75.0 & 90.0 & 75.0 & 60.0 & 50.0 & 50.0 & 50.0 \\
\hline $11: \mathrm{DRD2}$ RAT $\mid \mathrm{P} \ldots$ & 100.0 & 95.0 & 75.0 & 95.0 & 80.0 & 95.0 & 95.0 & 75.0 & 75.0 & 75.0 & & 95.0 & 75.0 & 95.0 & 60.0 & 55.0 & $55 . \theta$ & 55.8 \\
\hline 12:DRD3_RAT|P.. & 95.0 & $100 . \ominus$ & $75 . \theta$ & 95.0 & 85.0 & 95.0 & 100.0 & 75.0 & 75.0 & 75.0 & 95.0 & & 75.0 & 100.0 & 60.0 & 55.8 & 55.0 & 55.0 \\
\hline $13:$ DRD4_RAT|P... & 75.0 & 75.0 & 95.0 & 75.0 & 80.0 & 75.0 & 75.0 & 90.0 & 90.0 & 90.0 & 75.8 & 75.0 & & 75.0 & 60.0 & 50.0 & 50.0 & 50.0 \\
\hline 14:3PBL.A & 95.0 & 100.0 & 75.8 & 95.0 & 85.0 & 95.0 & 100.0 & 75.8 & 75.0 & 75.0 & 95.0 & 100.0 & 75.0 & & 60.0 & 55.8 & 55.0 & 55.8 \\
\hline $15: 4 B V N . A$ & 60.0 & 60.0 & 60.0 & 60.0 & 65.0 & 60.0 & 60.0 & 60.0 & 60.0 & 60.0 & 60.0 & 60.0 & 60.0 & 60.0 & & 70.0 & 70.0 & 70.0 \\
\hline 16:DRD1_HUMAN.... & 55.0 & 55.0 & 50.0 & 55.0 & 50.0 & 55.0 & 55.0 & 50.0 & 50.0 & 50.0 & 55.0 & 55.0 & 50.0 & 55.0 & 70.0 & & 100.0 & 95.0 \\
\hline 17:DRD1_DANRE... & 55.0 & 55.0 & 50.0 & 55.0 & 50.0 & 55.0 & 55.0 & 50.0 & 50.0 & 50.0 & 55.0 & 55.0 & 50.0 & 55.0 & 70.0 & 100.0 & & 95.0 \\
\hline 18:DRD1_RAT|P... & 55.0 & 55.0 & 50.0 & 55.0 & 50.0 & 55.8 & 55.0 & 50.0 & 50.0 & 50.0 & 55.8 & 55.0 & 50.0 & 55.0 & 70.0 & 95.0 & 95.0 & \\
\hline
\end{tabular}


Three dimensional reconstruction models of zebrafish DRD2(a-c) and DRD4(a-c)
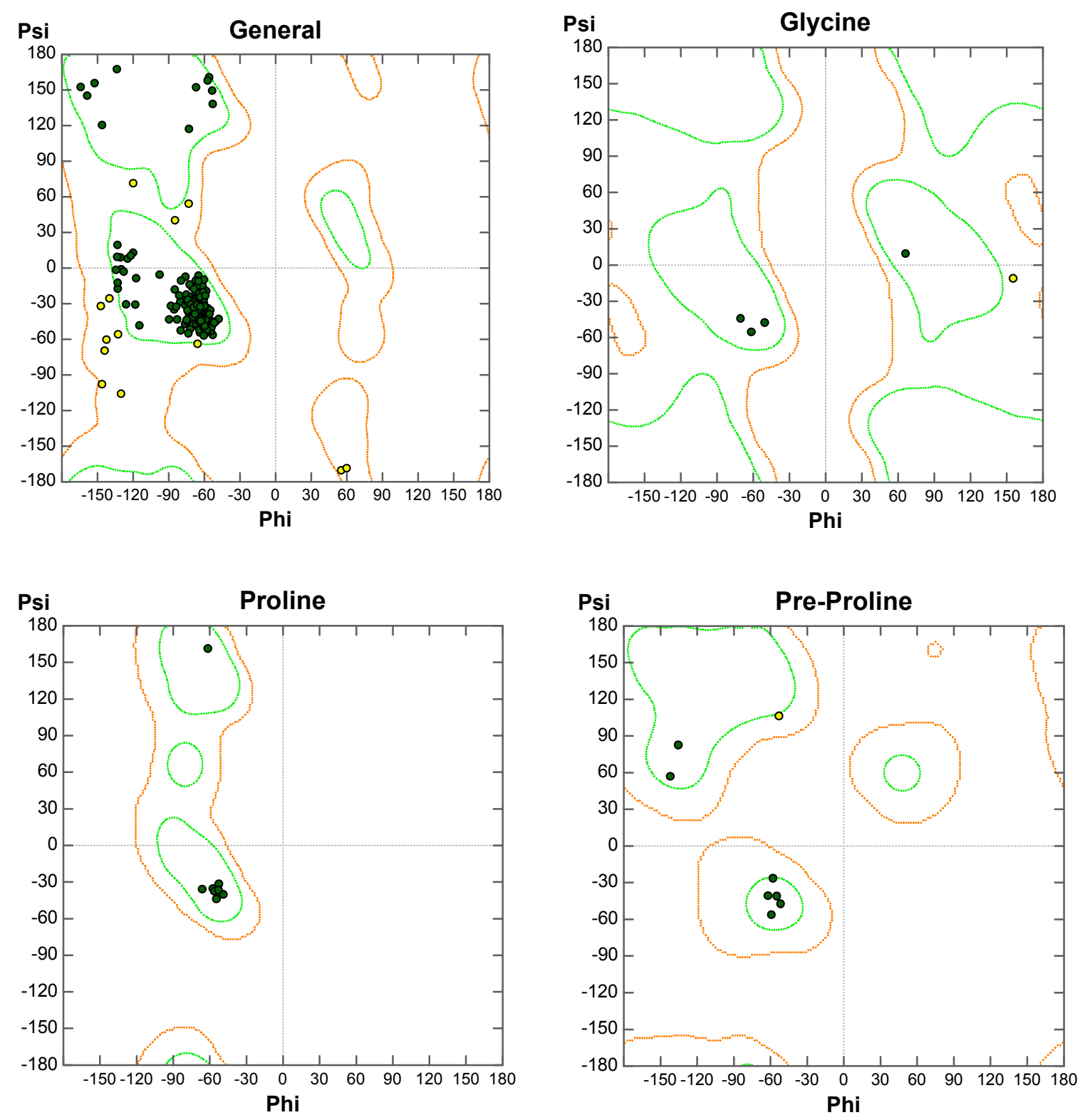

Figure 2s. Ramachandran plots for glycines, prolines, pre-prolines and for general residues of the selected zebrafish dopamine $\mathrm{D}_{2}(\mathrm{drd} 2 \mathrm{a})$ homology model. The contours indicate allowed (orange) and core (green) regions of $\varphi$ and $\Psi$ angles, and the filled green rings indicate amino acids within the core regions. The yellow rings indicate allowed regions. No outliers are present. 


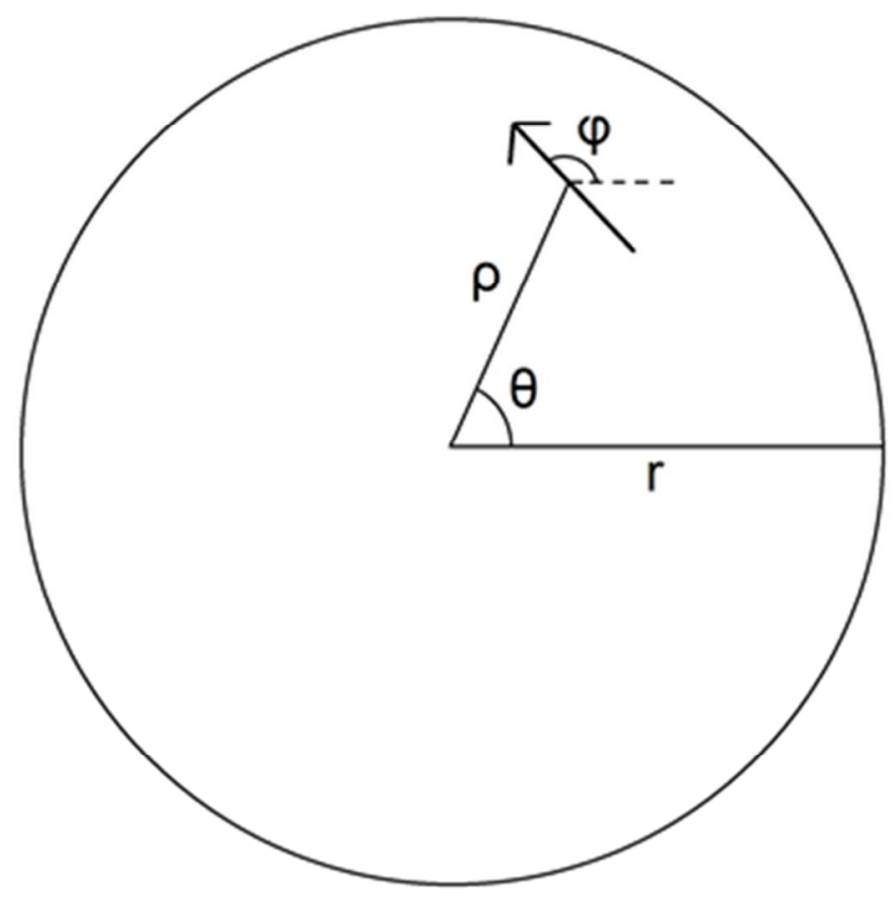

Figure 3s. Definition of radial distance, Rho $(\rho)$ and angles Theta $(\theta)$ and Phi $(\varphi)$.

\section{Definitions}

\section{Bout}

Zebrafish larvae swim by performing discrete bouts of activity that are well-defined and precisely quantifiable. A swim bout is here defined as swim events with more movement than a certain threshold (0.02 pixels/ frame). The distance the zebrafish larvae swim is calculated by three time resolutions (48, 24, and 12 frames) and all three must result in a movement above the threshold for the event to be treated as a bout.

\section{Turn}

A turn is defined as the total angular change in phi $(\varphi)$ in a bout.

\section{Definitions of behavioral parameters}

\section{Distance travelled}


The distance per minute averaged over the time window. Calculates the total distance the zebrafish has swum divided by the length of the recording (in minutes).

\section{Bouts per min}

The total number of bouts executed per minute averaged over the time window.

\section{Bouts}

The number of bouts performed within a time window. The zebrafish will be counted as swimming when in a swim bout and at a standstill when not moving.

\section{Standstills_T1-T2 (5-10 s, 10-15 s and 15-30 s)}

The number of periods the zebrafish has been at a standstill for the time interval T1-T2. The zebrafish is treated as standing still when not performing a bout.

\section{Bout distance}

The distance the zebrafish has swum per swim bout averaged over all swim bouts in the time window.

\section{Bout Duration}

The time that the zebrafish has swum per swim bout averaged over all swim bouts in each time window.

\section{Bout mean speed}

The average zebrafish swim speed during a bout averaged over all swim bouts in the time window.

\section{Bout max speed}

The zebrafish's maximum speed during a bout averaged over all swim bouts in the time window.

\section{Bout mean acceleration}

The average acceleration for the zebrafish's swim bout averaged over all swim bouts in the time window.

\section{Bout max acceleration,}

Th maximum acceleration for fish swim for a bout averaged over all swim bouts in the time window. 


\section{Center/wall preference (Center/Periphery 0-R1, R1-R2, R2-R3 or R3-R4)}

How much time the zebrafish spend in the center compared to the periphery of the well. There are four radial segments of the same area, which is defined as annuli with inner radius $\mathrm{R}$ and outer radius $\mathrm{R}, \mathrm{n}+1$ (except the center area is a circle of radius $\mathrm{R} 1$ ). Since the areas are to be equal, then the radii R1-R4 are $0.5 \mathrm{r}, 0.71 \mathrm{r}, 0.87 \mathrm{r}$, and $\mathrm{r}$, where $\mathrm{r}$ is the well radius. The value is the fraction of time the zebrafish has been in each area (all four parameters thus always sum to one).

\section{Distance from center (MeanRhoPos)}

The distance from the zebrafish to the center of the well per bout averaged over all bouts in a time window.

\section{Change in distance from center (RhoPosChange)}

How much the distance has changed in absolute value between the zebrafish and center of the well per bout and averaged over all bouts in a time window.

\section{Small and Large angle turn (Turn1 or 2)}

The number of turns below (Turn1) or above (Turn2) $60^{\circ}$. The number of turns is then calculated and normalized so that Turn $1+$ Turn $2=1$.

\section{Average rotational speed}

The zebrafish rotational speed using Phi $(\varphi)$. Calculated similar to MeanSPD.

Phi angle changeThe total change in the absolute value of Phi $(\varphi)$ during the time window, It is calculated per swim bout and averaged over all the bouts in the time window.

\section{Theta change}

The total change in the absolute value of Theta $(\theta)$ during the time window. It is calculated per swim bout and averaged over all the bouts in the time window.

\section{Theta mean speed}

The zebrafish's average speed in $\theta$-direction (thus only the velocity component in $\theta$-direction counts). Calculated otherwise as MeanSPD.

\section{Theta max speed}


The same as ThetaMeanSPD but using the maximum velocity instead.

\section{RightRevPref}

The time the larvae swims in right turns divided by the sum of the time it swims in right and left turns (a number between 0 and 1). The zebrafish is assumed to swim in right turns when the angle between the body's directional vector and vector $\mathrm{r}$ (Figure 1) from the center to the zebrafish is. $\pi / 6<\theta-\varphi(\bmod 2 \pi)<\pi$

\section{RightOrLeft}

The relationship between the time the zebrafish swims to the right or left and total swim time. If the angles have an absolute value that is less than $\pi / 6$, the zebrafish is considered to be facing the wall and not performing a left or right turn.

To calculate the octant parameters, the areas below the wells are divided into nine zones with the same area. Eight of them are annular sectors with a sector angle of $\pi / 4$ and the ninth is the center sector (a circle with a radius that is one third of the well), thus the same area as each wedge.

\section{OctPos}

The percentage of time that the zebrafish remained in their "home base" throughout the time window.

\section{OctSwim}

The percentage of time the zebrafish has been swimming in their "home base" compared to the total swimming time.

\section{OctVis}

The percentage of time the zebrafish swim into their "home base" (visit it) compared to the total number of visits in all areas. The zebrafish is assumed to be visiting an area when it moves from one area to another.

For the parameters below the sum of the ratio for each sector and the ratio for the center area, is equal to 1 . 


\section{$\operatorname{Oct}(1-8) \operatorname{Pos}$}

The amount of time that the zebrafish spent in each annular sector reported as a ratio between each annular sector (1-8) and the total time.

\section{CenterPos}

The amount of time that the zebrafish spent in the center area reported as a ratio between the center and the total time.

\section{$\operatorname{Oct}(1-8)$ Swim}

The amount of time that the zebrafish swim in each annular sector reported as a ratio between each annular sector (1-8) and the total time.

\section{CenterSwim}

The amount of time that the zebrafish swim in the center area and the total time reported as a ratio between the center and the total time.

\section{$\operatorname{Oct}(1-8)$ Vis}

The ratio between the number of visits that the zebrafish make in each annular sector and the total number of visits in all sectors.

\section{CenterVis}

The ratio between the number of visits that the zebrafish make in the center area and the total number of visits in all sectors.

Definition of classified turns

Scoot: Total angle change $<15^{\circ}$. Also bouts with no turn is classified as scoot.

JBend: Total angle change in the interval $10^{\circ}$ to $80^{\circ}$. Most of the movement is done sideways. Fraction between total angle change and distance travelled have to be $>40^{\circ} / \mathrm{mm}$

CBend: Total angle change $>80^{\circ}$. The first angle change in the turn must be in the interval $80^{\circ}$ to $130^{\circ}$.

OBend: Total angle change $>80^{\circ}$. The first angle change in the turn must be $>130^{\circ}$.

Startle: Maximum angle change $>45^{\circ} /$ frame and maximum speed $>0,065 \mathrm{~m} / \mathrm{s}$. 
Routine turn: Turn that has not been classified as scoot, J-bend, C-bend or O-bend.

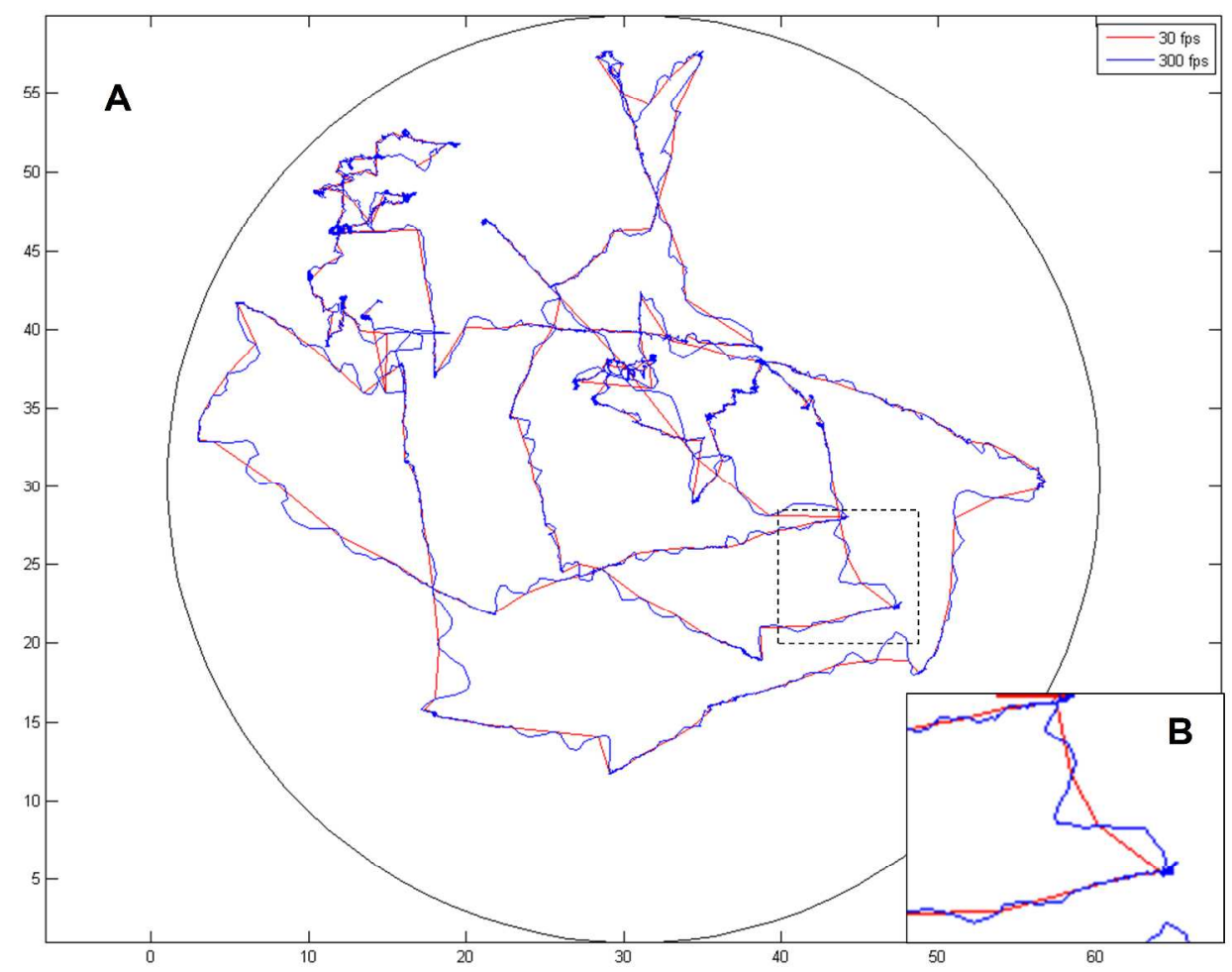

Figure 4s. (A) Trajectory pattern for the same video file analyzed using all frames (300 fps) or only $10 \%$ of frames (30 fps). Expansion (B) highlights a section of the trajectory pattern that demonstrates that an increased frame rate improves tracking of fast behavioral features. 


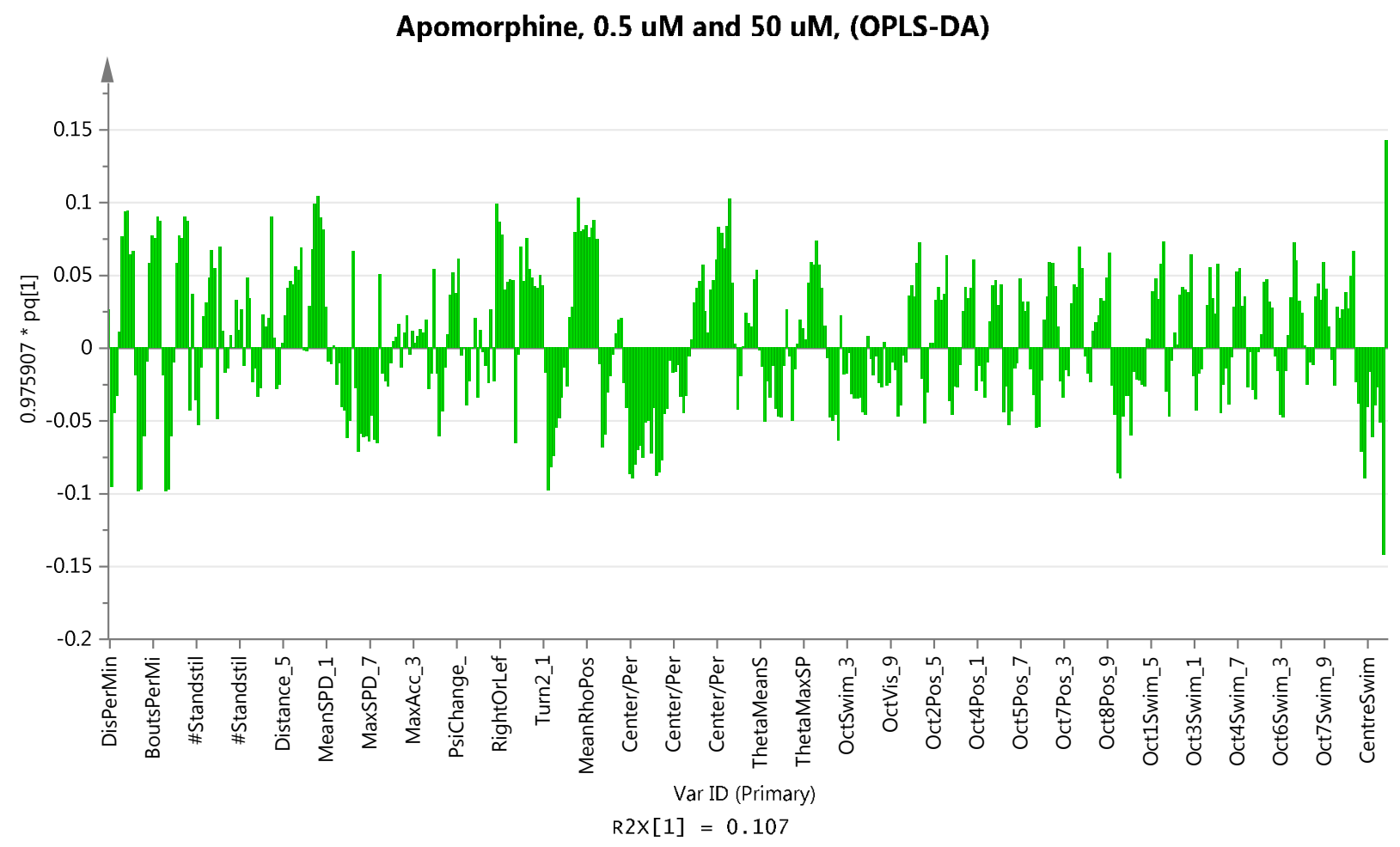

Figure 5s. OPLS-DA loading plots for all parameters separating groups treated with 50 and 0.5 $\mu \mathrm{M}$ in multivariate analysis.

\section{Wall preference (R3-R4)}

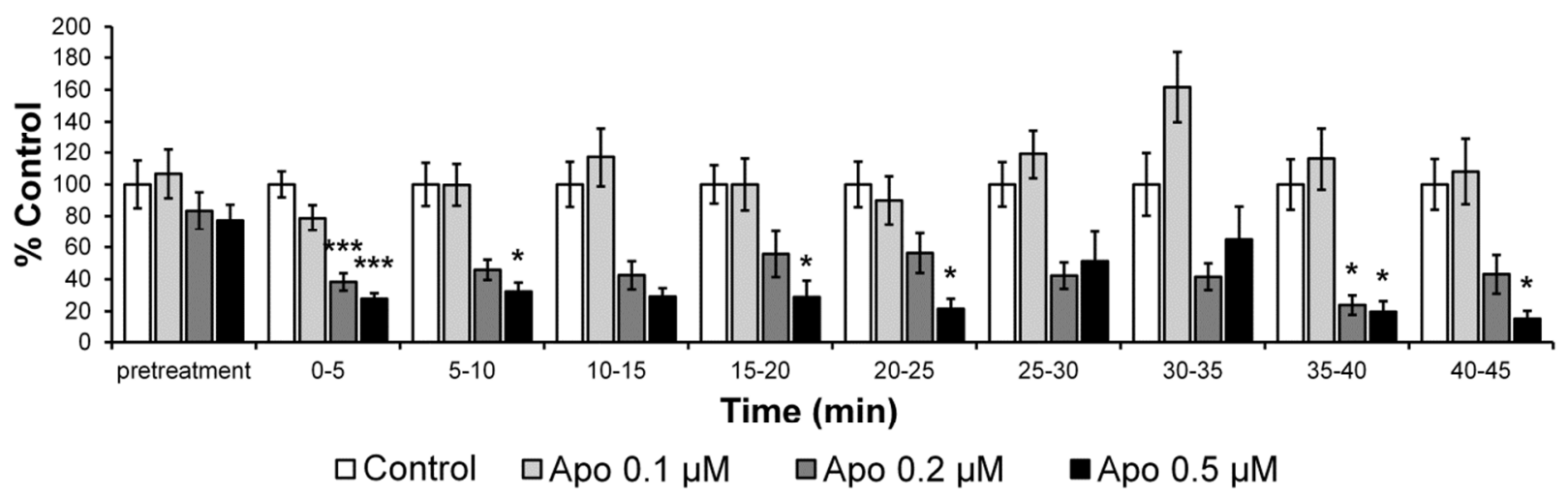

Figure 6s. Detailed analysis of wall preference (R3-R4) for the low-dose phenotype, $n=36$ (Control), $35(0.5 \mu \mathrm{M}), 35(0.2 \mu \mathrm{M})$ and $34(0.1 \mu \mathrm{M})$. Significance reported when comparing treatment with control groups: $* \mathrm{p}<0.05,{ }^{*} \mathrm{p}<0.001, * * * \mathrm{p}<0.0001$. Significance reported when comparing $0.5 \mu \mathrm{M}$ with $0.1 \mu \mathrm{M}$ : ${ }^{\dagger} \mathrm{p}<0.05$, ${ }^{+\dagger} \mathrm{p}<0.001$, †† $^{\mathrm{p}}<0.0001$. Bars represent mean over $5 \mathrm{~min} \pm \mathrm{SEM}$. 


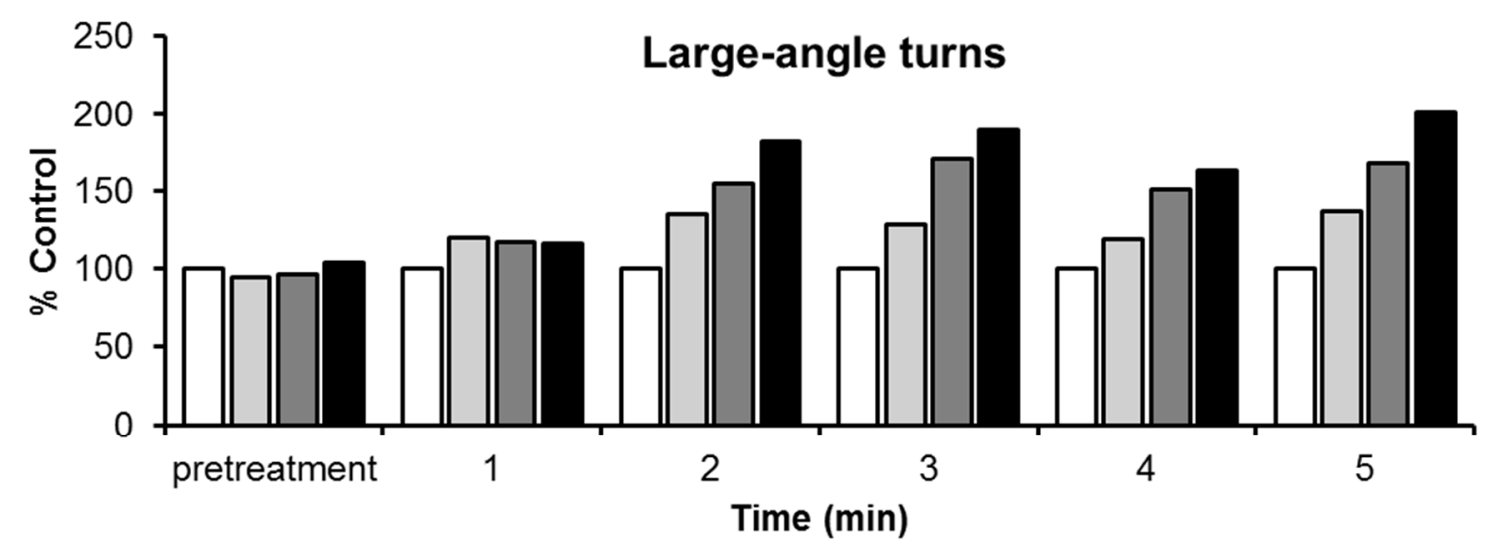

口Control Appo $0.1 \mathrm{uM}$ 口Apo $0.2 \mathrm{uM}$ 口Apo $0.5 \mathrm{uM}$

Figure 7s. Turn 2 after low dose apomorphine using 1 min time frames for the first $5 \mathrm{~min}, \mathrm{n}=36$ (Control), $35(0.5 \mu \mathrm{M}), 35(0.2 \mu \mathrm{M})$ and $34(0.1 \mu \mathrm{M})$.

A

Distance travelled (zebrafish)

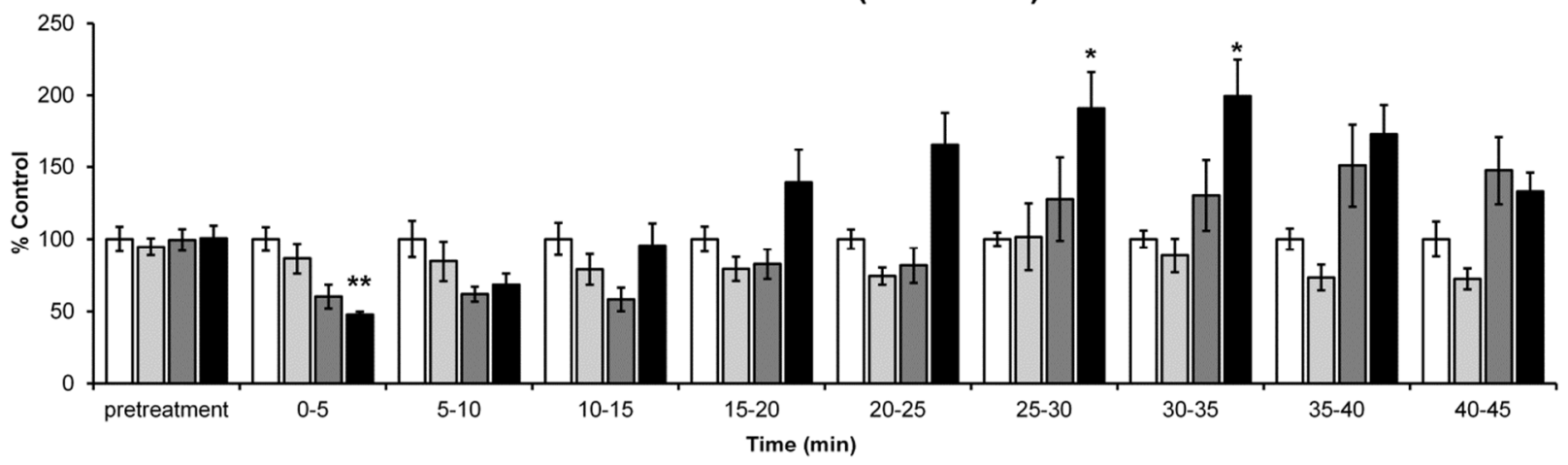

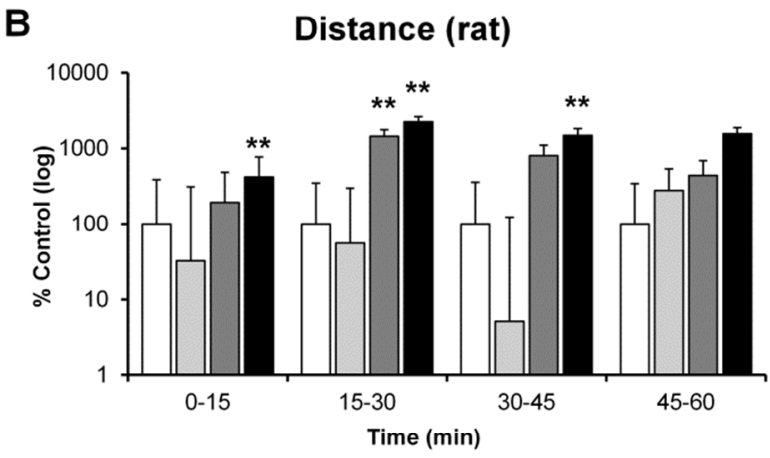

口Control $\square$ Apo $0.12 \mathrm{mg} / \mathrm{kg} \quad \square$ Apo $0.37 \mathrm{mg} / \mathrm{kg} \quad$ apo $1.1 \mathrm{mg} / \mathrm{kg}$

\section{Apomorphine (subcut) in humans}

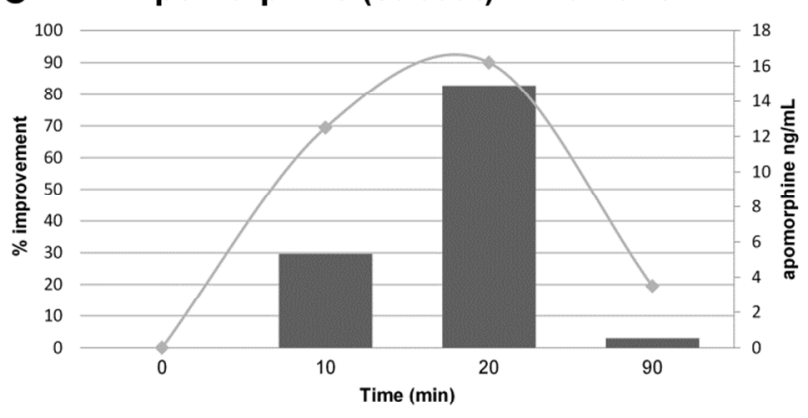

UPDRS Motor score improvement for apomorphine compared to placebo (\%)

$\rightarrow-$ Plasmaconcentration after apomorphine $(3 \mathrm{mg})$ administration $(\mathrm{ng} / \mathrm{ml})$

Figure 8s. Treatment with apomorphine increases locomotor activity in zebrafish (A), rats (B), and humans $(\mathrm{C})$ in an analog way. 